\title{
Double Faces of the Human Gut Microbiota
}

\author{
Siyi Zheng \\ College of Biology, Hunan University, Changsha 410082, China \\ Corresponding author email: zhengsiyi1204@163.com
}

Keywords: The gut microbiota; Immune adjustment; Nutrition metabolism; Obesity.

\begin{abstract}
The human intestine is colonized with symbiotic microbes, the gut microbiota has gained extensive attention. Recent researches have showed that the complex microflora has cohesive relationship with human health and the metabolism can be both beneficial and detrimental. The intestinal microbes can be concerned with its host through affecting the immune system, nutrition, metabolism, as well as inflammation or diseases. In this review, we discuss some current knowledge of the influence and function of the gut microbiota which might have therapeutic implications. In the aspects of absorbing nourishment from indigestible carbohydrates or regulating immunity system to defend pathogen, the gut microbiota is of great importance. In turn, any dysbacteriosis can bring about untoward effect or even diseases like obesity or gut inflammation. As the process and interaction between the host and the gut microbes are dynamic and complicated, it requires further researches to clarify the mechanism.
\end{abstract}

\section{Introduction}

The mucosal surfaces of human beings are colonies of a greatly large and dynamically complicated collection of microorganisms, almost $90 \%$ of our cells are microbial, whereas the remaining $10 \%$ belong to human beings [1]. And the microbial community in the intestine seems to be the most essential one, which contains the vast majority of the gut microbiota in human beings is an endogenous metagenome containing at least 100 times as many genes as human genome.

Recently the gut microbiota has received wide range of attention as it contributes in regulating and controlling several metabolic pathways of its host, such as nutrition absorption and immunity regulation. The development of the gut microbiota can be complexly affected by many factors like the environment, diet, drug, and life style. It plays critical roles in defining the myriad features of human biology.

The gut microbes show enormous importance in absorbing nutrition, especially it's essential for processing dietary polysaccharides [2], which are otherwise indigestible. As the gut microbes can be regarded as a metabolically active organ which can conduct multiple biochemical reactions, the host, under this circumstance, achieves an extent of metabolic adaptability to deal with changes in nutrition availability. However, while helping their host to extract calories from indigestible polysaccharides, the disorder or other changes in gut microbial ecology may affect the energy homeostasis, and might act as an important factor in causing obesity. There are differences in the gut microbes between obese and lean individuals, and the differences may cause different efficiency in extracting or storing energy from the given diet and finally, result in excess caloric intake which is one of the root cause of obesity [3].

In many researches, it has now been considered that the commensal bacteria especially the gut microbes play a significant role in the immunity, like sometimes through interacting with gut epithelium [4]. On the other hand, the gut microbes can also become the antigenic stimulus for inflammation [4]. As the relationship between the symbiotic microflora and the immune system is dynamic and precarious, great changes in the gut microbial ecology may become the pathogenesis.

Besides, the gut microbes can also help prevent and cure some disease [5], or even contribute to resist the cold[6]. As it means that a harmonious relation between microbiota and the host may benefit

metabolic homeostasis. Nevertheless, once the commensal microbiota community become 
maladjustment, it would affect the common metabolic function and might result in specific disease such as obesity[3], inflammation[4], or even neurodegenerative diseases[5], specific microflora can also be harm to the body, such as the fatty liver disease caused by high-alcohol-producing Klebsiella pneumoniae8.

\section{Benefit}

The human endogenous gut microflora is essential in absorbing nourishment, regulating epithelial development, and introducing congenital immunity. There are enormous uncultivated species and novel microorganisms which have been discovered in multiple colonic mucosal sites and feces[9] and the microbial diversity can help researchers exploring its roles in health and essential functions of these symbiotic bacteria include protecting against the injury of epithelial cell [10], regulating host fat storage[2], and stimulating intestinal angiogenesis [11].

\subsection{The Immune System}

As an important immune organ, the gut immune system has a challenge of resisting pathogens while holding unresponsiveness to food antigens and commensal microflora. It is evident that the gut invests abundant lymphoid tissue and immune cells to protect itself. The single layer of gut epithelium is the primary cellular barrier in preventing from exogenous infection which has a surface area expanding to the order of $400 \mathrm{~m} 2$, and each cell maintains a tight association with its neighbors and seals the whole surface of the gut. The barrier is a highly dynamic structure that at some extent limits antigens from entering the tissues, while the immune system constantly samples antigens.

There is abundant evidence shows that the normal commensal flora can result in an anti-inflammatory influence and is able to protect epithelial cells from toxic insult. It seems that mutualism appears to exist between the gut epithelium and the commensal flora which can maintain the integrity of epithelial. For example, the function of gut barrier can be increased through recognitions of TLR2 (toll-like receptor 2) and TLR9 (toll-like receptor 2) ligands by epithelial cells.[12] And the normal flora also induces cytoprotective proteins hsp25 and hsp72 in colonic epithelial cells.[13] Although in vitro[14] epithelial proinflammatory exists as the responses to the commensal flora, most individual maintain incurring disease with a tremendous intestinal flora. There also have interactions of commensals with the mucosal immune system. The evidence showed that it's sufficient to restore the mucosal immune system in germ-free mice through the reconstitution with a microbial flora. [15]

Over recent years, the use of probiotics has increased as they are believed to have beneficial effects to improve immune functions. Stainslaw et al. [16] have found that Lactobacilli are highy effective at surpressing virus-induced inflammation and can protect against lethal disease when targeted to the respiratory epithelium. The priming with live Lactobacilli resulted in diminished granulocyte recruitment and expression of multiple proinflammatory cytokines, including CXCL10, CXCL1, CCL2 and THF, additionally reduced virus recovery. Meanwhile, they have identified and characterized an effective innate immune shield mediated by Lactobacilli has a possibility to be ultimately served as critical and long-term protection against infection in the absence of specific antiviral vaccines.

Banasiewicz et al.'s research [17] showed that long-term use of probiotics Lactobacillus and Bifidobacterium has a Prophylactic effect on the occurrence and severity of Pouchitis. They did this experiment to assess the impact of a long-term use of the composite probiotics in patients after restorative proctocolectomy and after 9 months of probiotic taking, the number of patients and the average severity of pouchitis evidently decrease. As a result, they claimed that long-term use of probiotics might be a safe and well accepted way to prevent pouchitis. Similarly, Anna Berggren et al.[18] also study the effect of Lactobacilli in immune system, especially the defence against viral infections. In conclusion, the intake of two probiotic strains (DSM 15213 and DSM 13434) can authentically reduce the risk of acquiring common cold infections. 
Brylee A. Haywood et al.'s research [19] focused on infections in athletes, in such a special community, the attenuation of the number and severity of infection plays an important role. Through a randomised control trial with two arms, which are placebo and probiotic, they have found that the use of probiotics can efficiently reduce the duration and incidence of infections, while has no effect in the severity.

\subsection{Metabolism}

At early ages, humans have already developed symbiotic relationships with microbes [20]. Many factors affect the convergence of microbes in our guts, such as the environment, diet, genetics and temporal variation. The symbiotic microflora in our bodies can be regarded as a previously unknown organ in terms of its effects, especially its extensive metabolic capabilities. The microflora provides its host with a range of otherwise inaccessible metabolic capabilities [21].

The microbiome is relatively plastic, which is different from its host genome. It can be affected by diet, probiotics, drugs, and metabolites. Therefore, intentional alterations in the microbes can affect multiple biological processes and eventually impact the health, specific microbes have been viewed to have the ability to deactivate or activate specific xenobiotics and can alter the results of different therapeutic agents [22].

\subsection{Nutrient Absorption}

Among humans and other mammals, an enormous, complicated and dynamic community of microflora is coexisting with themselves. These microorganisms achieve energy particularly through carbohydrates, which are usually nondigestible to their host. Recently, the study of Bacteroides thetaiotaomicron [23] has led the research of the molecular mechanisms of the degradation of complex polysaccharides by the intestinal microbiota. The gut microbes can be collectively regarded as an active organ as they conduct a multitude of biochemical reactions which hold a critical post in nutrition. There is no need for the hosts to evolve such functions as the symbiotic microbes with metabolic capabilities can break down these compounds. The host can then adapt to changes in diet and nutrient availability. In return, the microbes can achieve a secure and nutrient-rich niche where they can multiply. Such arrangement makes a great deal of sense in commensalism.

Commensalism is a kind of interactions between gut bacteria and their host, and commensal relationships are concentrated on metabolic capabilities typically which allows either or both partners in this relationship to exploit an otherwise unavailable or poorly utilizable nutrient foundation. Intuitively, it seems that the host and the gut microbiota would be involved in competition for nutrients as their substrates both come from the diet by the host. However, the research showed that the microbiota could aid its host in extracting maximum nutritional value from the diet, as the germ-free raised animals require 30\% more caloric intake to maintain their body weight than the conventionally counterparts, who have traditional gut microbiota[24].

There might be complementary mechanisms working in it. The first one is that the microbial metabolism has the ability to converse many dietary substances into nutrients which can be utilized and absorbed by the host. And the second one is that the microbiota can alter the intrinsic metabolic machinery in host cells, and as a result, the nutrient uptake and utilization would be more efficient.

\section{Harm}

\subsection{Obesity}

Obesity is a disease across different populations with a complex etiology and variable prevalence. Obesity can be influenced by diet, behavior, environment, genetic factors, and as well as sex, age, race, ethnicity and socioeconomic status. Recently many studies have reported that there are differences in composition of the gut microbiota between obese and lean humans.

The microbial genomes encode metabolic capacities which their host haven't evolved wholly on their own, and these include the abilities to degrade indigestible components in the diet, and therefore affect the energy balance. The alteration by changes in gut microbial ecology gives small and 
sluggish changes in energy balance rather than having great contribution to obesity, and can result in magnificent changes in body weight over the course of a year [25]. The gut microbiota can be regarded as an additional contributing factor to the pathophysiology of obesity.

Peter J. Turnbaugh et al.'s research showed that obesity is related to changes in the relative abundance of two dominant bacterial colonies, the Bacteroidetes and the Formicutes [26]. They demonstrate that these changes can affect the metabolic potential of the gut microbiota through metagenomic and biochemical analyses. There results indicate that the obese microbiome can increasingly promote the ability to harvest energy from the diet.

This change refers to several linked mechanisms, such as the microbial fermentation of dietary polysaccharides, intestinal absorption of monosaccharides and short-chain fatty acids and the conversion in the liver to more complex lipids, and microbial regulation that promote deposition of the lipids in adipocytes of host genes [27].

Such findings can lead us to view that the obese microbiota can be more efficient than lean microbiota at extracting energy from a stated diet.

\subsection{Drug Toxicity}

The gut microbiota participates in the metabolism of many medical drugs, and can result in individual difference in drug efficiency and toxicity. Michael Zimmermann et al.'s research[28] combined gut commensal genetics with gnotobiotics to build a pharmacokinetic model for measuring metabolism of the nucleoside analog brivudine (BRV) across tissues, as to predict microbiome contributions to systemic drug and metabolite exposure. They found that BVU (hepatotoxic bromovinyluracil) will be concentrated in liver in those mice who have complicated microflora than germ-free mice, which may demonstrate that microbiota could be an enabling factor of drug toxicity.

A quantitative understanding of microbial factors which determine microbiome contributions can be conducive to explain interpersonal variability in drug response and provide possibilities for personal medical treatments.

\subsection{Gut Inflammation}

The ability of the gut microbiota to differentiate pathogenic and harmless microflora seems to break down in the developed world as the chronic inflammatory disease of the gut commonplace has increasingly occurred sometimes without overt infections. The development of gut inflammation and allergy is critically related to the homeostasis between gut antigens and host immunity [4]. The problem is that inflammation without infection is far more common than ever before as the result is that infectious diseases of the gut are mostly under control while gastrointestinal food allergies and idiopathic inflammatory conditions have come up more common. One explanation of this situation might be that the commensal flora can perform as an antigenic stimulus for the gut inflammation. As the relationship between the immune system and the commensal flora is precarious, any unrest in immune or epithelial homeostasis can lead to inflammation. Under such circumstance, the commensal flora seems to act as a surrogate bacterial pathogen, which is thought to be lifelong as the host response is incapable to eliminate the flora. There are experiment models which can prove that immune responses to the flora can cause IBD(Inflammatory bowel disease), for example, in human leukocyte antigen-B27(HLA-B27) transgenic rats, monoassociation with Bacteroides vulgatus can induce colitis, while Esherichia coli elicit no lesions[29].

\section{Conclusion}

The gut microbiota has intimate relationship with its host. On the one hand, the gut microflora helps its host to achieve nutrition, especially from those indigestible carbohydrates, and plays an important role in immune system while defending extraneous or intraneous pathogen, or it can even help maintaining body temperature during cold exposure by benefiting metabolic homeostsais. But however, on the other hand, dysbacteriosis can result to detrimental consequences, such as obesity, drug toxicity, and maybe diseases seem have no connection to the intestinal microbiota, like alcoholic 
fatty liver or Parkinson. The metabolic mechanism between the gut microbes and their hosts is so complicated that it could not be simply defined as beneficial or harmful. As a dynamic process, the complexity on variety and mechanism of the gut microbes remains more researches and has potential prospects, such as personalized medicine. Although there are many effects to be researched, the impact of gut microbes on human health and happiness is crucial and will be of great significance.

\section{References}

[1] Savage DC. Microbial ecology of the gastrointestinal tract [J]. Annual Review of Microbiology, 1977, 31:107-133.

[2] Backhed F, et al. The gut microbiota as an environmental factor that regulates fat storage [J]. PNAS, 2004, 101(44): 15718-15723.

[3] Ruth E.Ley, Fredrik Backhed, Peter Turnbaugh, et al. Obesity alters gut microbial ecology [J]. PNAS, 2005, 102(31):11070-11075.

[4] Thomas T.MacDonald, Giovanni Monteleone. Immunity, Inflammation, and Allergy in the Gut [J]. Science, 2005, 1920(307).

[5] Zhenda Shi, Jun Zou, Zhan Zhang, et al. Segmented Filamentous Bacteria Prevent and Cure Rotavirus Infection [J]. Cell, 2019, 179: 1-15.

[6] Baoguo Li, Li Li, Min Li, et al. Microbiota Depletion Impairs Thermogenesis of Brown Adipose Tissue and Browning of White Adipose Tissue[J]. Cell Reports, 2019, 26: 2720-2737.

[7] Timothy R.Sampson, Justine W.Debelius, Taren Thron, et al. Gut Microbiota Regulate Motor Deficits and Neuroinflammation in a Model of Parkinson's disease [J]. Cell, 2016, 167: 1469-1480.

[8] Jing Yuan, Chen Chen, Jinghua Cui, et al. Fatty Liver Disease Caused by High-Alcohol-Producing Klebsiella pneumoniae[J]. Cell Metabolism, 2019, 30:1-14.

[9] Paul B.Eckburg, Elisabeth M.Bik, Charles N.Bernstein, et al. Diversity of the Human Intestinal Microbial Flora [J]. Science, 2005, 308(5728):1635-1638.

[10] Rakoff-Nahoum S, Paglino J, Eslami-Varzaneh F, et al. Recognition of Commensal Mocroflora by Toll-like Receptors Is Required for Intestinal Homeostasis[J]. Cell, 2004, 118: 229-241.

[11] Stappenbeck TS, Hooper LV, Gordon JI. Developmental regulation of intestinal angiogenesis by indigenous microbes via Paneth cells [J]. PNAS, 2002, 99(24):15451-15455.

[12] E. Cario, G. Gerken, D. K. Podolsky. Toll-like receptor 2 enhances ZO-1-associated intestinal epithelial barrier integrity via protein kinase C [J]. Gastroenterology, 2004, 127(1): 224-238.

[13] K. Kojima et al. Enteric flora and Iymphocyte-derived cytokines determine expression of heat shock proteins in mouse colonic epithlial cells [J]. Gastroenterology, 2003, 124(5): 1395-1407.

[14] D. Haller, Lisa Holt, Sandra C.Kim, et al. Transforming Growth Factor- $\beta 1$ Inhibits Non-pathogenic Gramnegative Bacteria-induced NF- $\kappa$ B Recruitment to the Interleukin-6 Gne Promoter in Intestinal Epithelial Cells through Modulation of Histone Acetylation[J]. The Journal of Biological Chemistry, 2003, 2787(26): 23851-23860.

[15] Y. Umesaki, Y. Okada, S. Matsumoto, et al. Segmented Filamentous Bacteria Are Indigenous Intestinal Bacteria That Activate Intraepithelial Lymphocytes and Induce MHC Class II Molecules and Fucosyl Asialo GM1 Glycolipids on the Small Intestinal Epithelial Cells in the Ex-Germ-Free Mouse[J]. Microbiol. Immunol, 1995, 39(8): 555-562. 
[16] Stain J.Gabryszewski, Ofir Bachar, Kimberly, et al. Lactobacillus-Mediated Priming of the Respiratory Mucosa Protects against Lethal Pneumovirus Infection [J]. The Journal of Immunology, 2011, 186:1151-1161.

[17] Banasiewicz Tomasz, Stojcev Zoran, Walkowiak Jaroslaw, et al. Long-Term Use of Probiotics Lactobacillus and Bifidobacterium Has a Prophylactic Effect on the Occurrence and Severity of Pouchitis: A Randomized Prospective Study [J]. BioMed Research International, 2014,208064.

[18] Anna Berggren, Irini Lazou Ahren, Niklas Larsson, Gunilla Onning. Randomised, double-blind and placebo-controlled study using new probiotic lactobacilli for strengthening the body immune defence viral infections [J]. Eur J Nutr, 2010.

[19] Brylee A.Haywood, Katherine E.Black, Dane Baker, et al. Probiotic supplementation reduces the duration and incidence of infections but not severity in elite rugby union players [J]. Journal of Science and Medicine in Sport, 2014, 17:356-360.

[20] Dominguez- Bello MG, Blaser MJ, Ley RE, et al. Development of the human gastrointestinal microbiota and insights from high - throughput sequencing [J]. Gastroenterology, 2011, 140(6): 1713-1719.

[21] Qin J, Li R, Raes J, et al. A human gut microbial gene catalogue established by metagenomic sequencing [J]. Nature, 2010, 464: 59-65.

[22] Haiser HJ, Turnbaugh PJ. Is it time for a metagenomic basis of therapeutics [J]? Science 2012, 336: $1253-1255$.

[23] Lora V.Hooper, Tore Midtvedt, Jeffrey I.Gordon. How host-microbial interactions shape the nutrient environment of the mammalian intestine [J]. Annu.Rev.Nutr, 2002, 22:283-307.

[24] Wostmann BS, Larkin C, Moriarty A, Bruckner Kardoss E. Dietary intake, energy metabolism, and excretory losses of adult male germfree Wistar rats [J]. Lab Anim. Sci, 1983, 33(1): 46-50.

[25] Legal, K. M., Troiano, R. P. Changes in the distribution of body mass index of adults and children in the US population [J]. International Journal of Obesity, 2000, 24(7): 807-818.

[26] Peter J.Tumbaugh, Ruth E.Ley, Michael A.Mahowald, et al. An obesity-associated gut microbiome with increased capacity for energy harvest [J]. Nature, 2006, 444.

[27] Backhed, F. et al. The gut microbiota as an environmental factor that regulates fat storage [J]. Proc. Natl Acad. Sci, 2004, 101: 15718-15723.

[28] Michael Zimmermann, Maria Zimmermann-Kogadeeva, Rebekka Wegmann, Andrew L.Goofman. Separating host and microbiome contributions to drug pharmacokinetics and toxicity [J]. Science, 2019, 363.

[29] G. Bouma, W. Strober. The Immunological and Genetic Basis of Inflammatory Bowel Disease [J]. Immunology, 2003, 3: 521-533.

[30] Maggie A. Stanislawski, Dana Dabelea, Leslie A.Langle, et al. Gut microbiota phenotypes of obesity [J]. Biofilms and Microbiomes. 2019, 18. 